\title{
How to Write a Manuscript: Tips for the Young Researchers
}

\author{
Prosenjit Ghosh ${ }^{1}$, Anshuman Gogoi ${ }^{2}$
}

\begin{abstract}
Many journals and other published guidelines provide large, sometimes strict instructions to be included for becoming eligible in academic journals. However, there is a lack of simple and clear recommendations on how to write such scientific articles. We propose a simple and easy-to-follow hypothesis-based approach. The introduction section should start by introducing the relevant concepts, formulate the study hypothesis, mention the gap in knowledge in the existing literature, and describing the study hypothesis in simple language at the end. The material and methods section must describe the study design, sample or material, the tools, instruments, inclusion and exclusion criteria, and procedures used to test the study hypothesis. The results section must describe the data analyses that lead to the confirmation or rejection of the hypothesis. The discussion must state whether the study hypothesis has been confirmed or rejected and compare the study result with previous research. It should clarify the limitations of the study and explore the relevance of the study findings for clinical practice and future research. An abstract at the beginning of the manuscript, usually structured with objectives, material and methods, results and conclusion, should be added. Acknowledgments, ethical approval, informed consent by study subjects, conflicts of interests by authors, and a reference list will be needed in most scientific journals.

Keywords: Hypothesis, Manuscript, Medical journal, Scientific paper.

Indian Journal of Private Psychiatry (2021): 10.5005/jp-journals-10067-0073
\end{abstract}

\section{INTRODUCTION}

The role of abstract is immense as most of the scientific community will read the full paper only if the quality of the abstract is found good. The abstract should be ideally within 200-250 words. The keywords should be at least three in number and represent the core theme of the manuscript. Publication of research is not only essential for the growth of scientific knowledge but it has become mandatory eligibility criteria for professional growth in terms of promotion for faculty members of medical colleges in India. ${ }^{1}$ So it has become mandatory for all the faculty members to learn how to write a manuscript and get it published in indexed journals.

For the new authors, a simple hypothesis-based approach is easier; the authors will formulate a study hypothesis in the background of present knowledge and will try to establish the hypothesis with appropriate study design and tools. Manuscripts are usually prepared in five sections-background, methodology, results, discussion, and conclusion. There are other sections of manuscripts, like abstract; the conflict of interest statement by the researchers; acknowledgment of substantial contributions of people, who are not listed as authors; ethical committee permission; and informed consent by the study subjects.

The introduction has four main areas-approach, conception, justification, and hypothesis.

- Approach: Initially the author should describe the disorder/ concept/behavior to be investigated.

- Concept: Clear idea about the disease/condition being investigated.

- Justification: The authors should explain the specific reasons why they want to investigate the diseases/concepts/behavior. Such relevance could be diagnostic, new treatment modality, prevention and control of diseases, testing new drugs/vaccines, getting additional or new information, verifying existing information, explaining cause-effect relationships, public health program evaluation, and so on.
1,2Department of Psychiatry, Silchar Medical College and Hospital, Silchar, Assam, India

Corresponding Author: Prosenjit Ghosh, Department of Psychiatry, Silchar Medical College and Hospital, Silchar, Assam, India, Phone: +91 9435072563, e-mail:p_ghosh72@yahoo.com

How to cite this article: Ghosh P, Gogoi A. How to Write a Manuscript: Tips for the Young Researchers. Ind J Priv Psychiatry 2021;15(1):49-51.

Source of support: Nil

Conflict of interest: None

- Hypothesis: Hypothesis is a question related to a problem in the population that the investigator wants to find the answer by making an assessment/intervention in the study population. There are various stages to formulate the hypothesis or research question. Initially, it starts with a vague and loose idea/concept about a health topic and then narrows down to a concrete, researchable issue, frames problem in specific terms (clinical/ public health/epidemiological, etc.), focuses on one issue, and is written in everyday language; the research question should be related to the plausible actions that would be taken once the hypothesis is either rejected or confirmed.

Hypothesis is concerned with what the researcher wants to know. The researcher can take help of various sources while framing the hypothesis-review of work of others in the area of interest, attending research meetings/conferences, having a skeptical attitude about prevailing beliefs, careful observation, etc. A good hypothesis should be feasible (adequate sample size), interesting, novel (provides new information), ethical, and relevant (advances in scientific knowledge).

The review of existing literature is very important in formulating the hypothesis. The authors should clearly mention about the deficiency in knowledge/information about the study disease/

(o) The Author(s). 2021 Open Access This article is distributed under the terms of the Creative Commons Attribution 4.0 International License (https://creativecommons. org/licenses/by-nc/4.0/), which permits unrestricted use, distribution, and non-commercial reproduction in any medium, provided you give appropriate credit to the original author(s) and the source, provide a link to the Creative Commons license, and indicate if changes were made. The Creative Commons Public Domain Dedication waiver (http://creativecommons.org/publicdomain/zero/1.0/) applies to the data made available in this article, unless otherwise stated. 
condition and what new insight can be added by testing the study hypothesis. While formulating the hypothesis, the authors must define measurable exposures and outcomes.

Literature review may be divided into two parts-information seeking and critical appraisal.

- Information seeking: To search the existing literature by using various search engines with keywords to identify a group of useful and related articles, books, conference proceedings, etc.

- Critical appraisal: The ability to identify those studies that are relevant to the present study hypothesis and have a good level of evidence. Studies relevant to the present study topic should be included in the final review of the literature and analyze each study on the basis of study design, methodology, sample size, strength, and limitations/weaknesses. A good hypothesis should have one exposure/outcome and clearly defined sample/ variables and focuses on the primary objective.

The next step is to translate the research questions to objectives-should be framed in scientific/epidemiological terms; written in scientific/epidemiological language; only one verb should be used for each objective; may be divided into primary and secondary; has to be clear about the type of question: descriptive questions (measuring a quantity) and analytical/experimental questions (testing a hypothesis).

The research question: To improve the management of treatmentresistant schizophrenia, we want to know whether repetitive transcranial magnetic stimulation (rTMS) therapy at least for 6 weeks reduces psychopathology as compared to those who do not.

Primary objective: Determine the effect of rTMS therapy at least for 6 weeks on psychopathology in patients with treatment-resistant schizophrenia compared to those who do not.

\section{Materials and Methods}

This section will describe the tools, study design, study population, and data collection method used for finding the answers to the research question.

- Study setting: It should clearly mention the site of the study, like whether community setting or tertiary care setting, what was the duration of the study, and why the specific setting was chosen should also be explained.

- Study design: Making the right choice of the study design is of paramount importance. Various types of study design are qualitative studies or quantitative studies, observational studies or experimental studies, and retrospective studies or prospective studies.

- Sample selection: The sampling procedure (random sampling, systematic sampling, convenience sampling, presumptive sampling, etc.), sample size, the inclusion and exclusion criteria, and logical explanations of selecting such criteria must be recorded.

- Tools/instruments: The study instruments/protocols and interventions should be described and they must have high validity and reliability. The tools must be standardized and if needed necessary permissions for use should be obtained from the original author. Some tools may need translation into the local language; in such cases, they have to be retranslated back into the English language and validity to be assessed. It is important to mention whether such tools were used earlier in
India. It is also important to remember that some tools might need training before a researcher can administer them.

- Data collection: The data will be collected and statistical methods will be used to analyze the data.

\section{Informed Consent}

All the study subjects including the controls must give informed consent and should sign the consent form in the presence of a witness.

\section{Results}

A complete description of the study sample should be given, if any subjects dropped out of the study or developed any adverse events (drug trials) should be mentioned. The outcome of interventions/ exposures should be chronologically assessed and the results of such interventions discussed.

The appropriate use of tables and figures is included; they should be properly labeled and numbered and should not repeat the part of the text. Tables and figures help in understanding the results in a better way and should be used to highlight the main findings of the study.

The analysis of results must reach a conclusion whether the hypothesis is proved correct or it can be rejected.

\section{Discussion}

The discussion usually starts with the declaration that the answer to the research question is either affirmative or negative. ${ }^{2}$

Then the authors must analyze if the outcome of the study is in agreement with the findings of similar previous studies and there is evidence from past studies with a similar context of research. If the outcome of the study contradicts previous research findings, then a reasonable explanation has to be given by the authors.

If the outcome of the study hypothesis brings any novel findings, then the possible reasons must be mentioned. The author should try to mention a few of the Indian studies while comparing the findings of his/her study.

One important aspect of the manuscript is the limitations of the study - the sample (too small or not representative of general population), the data collection (recall bias in case of retrospective study), the study design (lack of a control group), and whether the final conclusion has any methodological errors, like confounding errors or effect modifiers. In other words, whether the results can be applicable in a wider perspective across geographical regions.

\section{Conclusion}

Conclusion should summarize the outcome of the study findings in terms of clinical, research, or policy implications. The findings can be used in clinical settings or in future projects with improved study design to get more specific answers.

\section{ACKnOWLedgments}

To acknowledge the support provided by persons outside the research team, providing logistic support, administrative help, or helping with the field work.

\section{Reference}

This list should be arranged in accordance with the instructions of the particular journal in which the author is planning to submit 
the research work. There are different ways to format citations. The most common style for medical journals is the Vancouver style.

\section{Conflict-of-interest Statement}

The credibility of scientific works depends mainly on minimum conflict of interest during various stages of decision-making after the initial submission by an author. Conflict of interest exists when the reviewer, referee, or editor has financial or personal interests that inappropriately influence (bias) his or her decisions. ${ }^{3}$

\section{Statement of Informed Consent}

Patients have a right to privacy that should not be violated without informed consent. No identifying information, including patients' names and photographs, should be published.

There are specific recommendations and checklists for different types of manuscripts, like for systematic reviews, PRISMA ${ }^{4}$ and RAMSTAR $^{5}$; for randomized trials, CONSORT; ${ }^{6}$ for observational studies STROBE7 ; and for clinical practice guidelines, AGREE. ${ }^{8}$

\section{Word Limits}

Many journals may mention about the word limits for various categories of manuscript. ${ }^{9}$ Authors should adhere to those guidelines regarding word limits, number of reference limits, etc. while preparing the manuscript.

\section{References}

1. Extraordinary, part III-Section 4. Gaz India 2020;66:1-9.

2. Heun R. How to write a scientific paper: a hypothesis-based approach. Glob Psychiatry 2018;1(1):3-6. DOI: 10.2478/gp-2018-0004.

3. Open J Psychiatry Allied Sci. Authors instructions. 2020. Guwahati, Assam. Available from: https://www.ojpas.com/author-instructions. html

4. Liberati A, Altman DG, Tetzlaff J, et al. The PRISMA statement for reporting systematic reviews and meta-analyses of studies that evaluate health care interventions: explanation and elaboration. J Clin Epidemiol 2009;62(10):e1-e34. DOI: 10.1136/bmj.b2700.

5. Kung J, Chiappelli F, Cajulis OO, et al. Systematic reviews to clinica recommendations for evidence-based health care: validation of revised assessment of multiple systematic reviews (RAMSTAR) for grading of clinical relevance. Open Dent J 2010;4:84-91. DOI: 10.2174/1874210601004020084

6. Schulz KF, Altman DG, Moher D, et al. CONSORT 2010 statement: updated guidelines for reporting parallel group randomized trials. Ann Intern Med 2010;152(11):726-732. DOI: 10.1136/bmj.c332.

7. Elm EV, Altman DG, Egger M, et al. Strengthening the reporting of observational studies in epidemiology (STROBE) statement: guidelines for reporting observational studies. BMJ 2007;335(7624):806-808 DOI: $10.1136 / \mathrm{bmj} .39335 .541782 . A D$

8. Brouwers MC, Kerkvliet K, Spithoff K, et al. The AGREE reporting checklist: a tool to improve reporting of clinical practice guidelines. BMJ 2016;352:i1152. DOI: 10.1136/bmj.i1152.

9. Indian J Psychiatry. Authors information. 2020. Delhi, India. Available from: https://www.indianjpsychiatry.org/contributors.asp 